\title{
Zoonosis con reservorios silvestres: Amenazas a la salud pública y a la economía
}

\author{
Carlos Cabello C 1 ,a, Felipe Cabello $C^{2}$. \\ Zoonoses with wildlife reservoirs: \\ A threat to public health \\ and the economy
}

The world is experiencing an increase in emergent infections as a result of anthropogenic changes of the biosphere and globalization. Global warming, unrestricted exploitation of natural resources such as forests and fisheries, urbanization, human migration, and industrialization of animal husbandry cause environmental destruction and fragmentation. These changes of the biosphere favor local emergence of zoonoses from their natural biotopes and their interaction with domestic animals and human populations. Subsequently, international commerce, human and animal migration and travel, favor the dissemination of these zoonotic pathogens worldwide. Chile is undergoing an important degradation of many wild-life biotopes, affecting their diversity and contributing to the dissemination of zoonoses such as Chagas disease, Hantavirus, rabies, fish tapeworms, and marine vibriosis. Moreover, agents of many other zoonoses such as leptospirosis, hydatidosis, salmonellosis, rabies, brucellosis and anthrax have been detected in different wild-life environments in the country. The intensification and accelerations of the anthropogenic deterioration of the biosphere in Chile, as results of the unrestricted utilization of natural resources and global climate change, suggests that emergence of new zoonoses in the near future will lead to important public health and economic problems. Forestalling of these problems will require active epidemiological surveillance of wild and domestic animals with a wide range of modern molecular and ancillary epidemiological tools. This also demands government and private sector (i.e., animal husbandry) intervention, funding and the collaboration of professionals in human and veterinary medicine with those in the environmental sciences including ecology, climatology and oceanography (Rev Méd Chile 2008; 136: 385-93).

(Key w ords: Ecology; Epidemiology; Zoonoses)

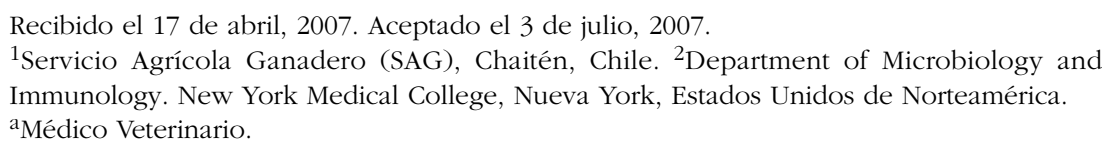

T a presencia de enfermedades emergentes ha Lonstituido un serio y dramático mentís al concepto utópico de la conquista definitiva de la

Correspondencia a: Dr. Felipe Cabello. Valhalla, NY 10895, U.S.A. Phone: 914 5944182. Fax: 914 5944176. E mail: cabello@nymc.edu enfermedad infecciosa ${ }^{1-3}$. La aparición de ellas ha dejado en claro deficiencias conceptuales y técnicas en el tratamiento de estos problemas por individuos, gobiernos y entidades internacionales responsables de la prevención y la solución de ellos 4,5 . Gran parte de las enfermedades emergentes, nuevas y antiguas, son zoonosis $4,6,7$ (Tabla 1). 
Tabla 1. Ejemplos de zoonosis emergentes con reservorio silvestrea

\begin{tabular}{|lllll|}
\hline Patógeno & Reservorio & Vector & Lugar & Año \\
\hline Cryptosporidium & roedores & ninguno & EE.UU. & 1980 \\
Hantavirus & roedores & ninguno & EE.UU. & 1996 \\
& & & Argentina & 1993 \\
Yersinia pestis & roedores & pulga & Chile & 1995 \\
EEV $^{b}$ & caballos & mosquito & India & 1993 \\
FHCC & ovejas, liebre & garrapata & Mexico & 1995 \\
Tifus & roedores & piojos & Bulgaria & 1994 \\
& & & Burundia & 1996 \\
Hendra virus & murciélagos & ninguno & Australia & 1995 \\
Ebola virus & roedores & ninguno & Congo & 2000 \\
& & & Sudan/Uganda & 1997 \\
Influenza H5N1 & patos, pollos & ninguno & Hong Kong & 1997 \\
Nipah virus & murciélago & cerdos & Malasia & 1999 \\
Influenza H9N2 & pollos & ninguno & Hong Kong & 1999 \\
Viruela del mono & monos & roedores & EE.UU. & 2003 \\
SRAS virus & murciélago & ninguno & China & 2003 \\
& comadrejas & & & \\
\hline
\end{tabular}

aTomado y modificado de referencia 53.

bVirus de la encefalitis equina venezolana.

El concepto de zoonosis implica que los patógenos causantes de la enfermedad tienen, en general, un reservorio animal silvestre, y de este reservorio animal generalmente asintomático, el patógeno puede ser transmitido directamente a humanos o a los animales domésticos, los que a su vez pueden transmitirlo al ser humano ${ }^{8-10}$, Los patógenos que generalmente causan zoonosis pueden ser bacterias, virus o parásitos ${ }^{8-11}$ y en algunas raras ocasiones hongos ${ }^{8-11}$. En la mayoría de ellas, la mantención de un reservorio silvestre aparece como una condición esencial de la persistencia de la zoonosis en una determinada área geográfica $^{8-13}$.

Más de la mitad (62\%) de los patógenos humanos son de origen zoonótico ${ }^{7,11,12}$ y como resultado de los cambios antropogénicos de la biósfera, los patógenos de origen animal constituyen el grueso de las enfermedades emergentes ${ }^{7,12-}$ 14. En esta revisión discutiremos, brevemente, algunos aspectos que explican la emergencia de las zoonosis con un reservorio silvestre como problemas de salud pública en el mundo y en Chile.
cVirus de la fiebre hemorrágica de Crimea y el Congo.

dVirus del Síndrome Respiratorio Agudo Severo.

FACTORES AMBIENTALES Y SOCIALES QUE INFLUYEN

EN LA EMERGENCIA DE PATÓgENOS ZOONÓTICOS SILVESTRES COMO PATÓGENOS HUMANOS

El concepto del reservorio silvestre de las zoonosis incluye también el concepto de biotopo ${ }^{8-11}$. Un biotopo corresponde a una comunidad de animales y plantas coexistiendo en una región geográfica con un paisaje y clima determinados y cuya estructura comunitaria asegura la persistencia del patógeno en la comunidad ${ }^{8-11}$. Las zoonosis son, entonces, enfermedades infecciosas que tienen nidalidad, esto es la habilidad de mantener un foco dinámico y permanente de circulación del patógeno en animales en un ambiente geográfico determinado $7,8,10,14$. Implícito en estos conceptos está el hecho que cualquier factor que tenga la capacidad de alterar el biotopo conteniendo el reservorio silvestre de una zoonosis, tiene el potencial de alterar su nidalidad, modificando de esta manera la epidemiología de la zoonosis ${ }^{7-11,15}$. La mayor parte de los cambios que modifican los equilibrios dinámicos de los reservorios animales 
silvestres de patógenos con potencial zoonótico son antropogénicos, (Tabla 2) 4,5,7,10,16, e incluyen una serie de cambios ambientales como el aumento de la población y la migración de ésta a lugares previamente inhabitados ${ }^{4-7,10}$. Estos cambios poblacionales se acompañan además de degradación ambiental, polución y cambios climáticos $4,5,12,17-$ 19. Por ejemplo, la resurgencia de varias fiebres hemorrágicas en América del Sur ha sido el resultado de la fragmentación y la destrucción de hábitats boscosos en Argentina (virus Junín), Bolivia (virus Machupo) y Venezuela (virus Guanarito) $)^{5,7-9,14}$. Similarmente, la destrucción forestal y cambios climáticos en Malasia, limitaron la nutrición y los hábitos alimentarios de los murciélagos de la fruta, resultando en el pasaje del virus Nipah de estos animales silvestres a los cerdos y de éstos últimos a seres humanos ${ }^{7-9,15}$. La intensificación de la crianza industrial de animales como cerdos y aves en China y en otros países del oriente, ha sido también responsable de la aparición de nuevas formas genéticas del virus de la influenza $\mathrm{A}^{11,20}$.

La invasión humana de nichos ecológicos silvestres también facilita la transmisión de patógenos de especies silvestres al ser humano, como al parecer sucedió con el virus del $\operatorname{SIDA}^{4,16,21}$ y los virus del grupo Ebola ${ }^{4,16,26,21}$. La destrucción de biotopos silvestres en muchas oportunidades es resultado de la urbanización que aumenta las posibilidades de exposición a patógenos, como ha sucedido en Estados Unidos de Norteamérica y en Europa con la enfermedad de Lyme y otras enfermedades transmitidas por garrapatas Ixo$\operatorname{des}^{8,9,22,23}$. La intrusión humana en el biotopo silvestre de la enfermedad de Lyme también ha aumentado su transmisión humana porque han disminuido los predadores, como zorros y coyotes $8,9,22,23$. Esto ha producido un aumento de la población del ratón de patas blancas (Peromyscus leucopus) que son excelentes huéspedes y el reservorio silvestre asintomático más importante de Borrelia burgdorferi en Estados Unidos de Norteamérica ${ }^{8,9,22,23}$.

La degradación de los biotopos silvestres conteniendo potenciales patógenos humanos secundaria a actividades humanas, en general, conlleva a una disminución de su biodiversidad que facilita la infección humana con estos patógenos en estos biotopos $^{2,7-9,15,22,23}$. Algunas actividades humanas, como el aumento del comercio y de los viajes, diseminan estos patógenos, sus vectores e incluso a sus mismos reservorios animales a través del globo $4,7,12,24-27$. En Estados Unidos de Norteamérica, la introducción del virus de la fiebre del Nilo Oeste por mosquitos Culex ha hecho que esta fiebre se haya diseminado a todo el país en menos de 10 años, creando reservorios autócto-

\section{Tabla 2. Factores asociados a la emergencia de zoonosis ${ }^{\mathrm{a}}$}

1. Cambios en los usos de terreno y agua y prácticas agrícolas: deforestación, crianza industrial de animales, urbanización, disrupción de nichos ecológicos, perdida de la biodiversidad

2. Cambios demográficos y sociales: aumento de la población, migración, pobreza, desnutrición, cambios en comportamiento sexual

3. Deficiente salud pública y vigilancia epidemiológica: humana y veterinaria, ausencia de control de la calidad de los alimentos

4. Cambios en hábitos alimenticios: ingestión de alimentos crudos (carne, pescados, verduras), ingestión de alimentos importados, uso de alimentos contaminados en la crianza industrial de animales, consumo de animales silvestres

5. Evolución de patógenos: mutación y recombinación que aumentan virulencia y amplían el rango de huésped; resistencia a antimicrobianos

6. Globalización: viajes, transporte de animales y alimentos, comercio global, introducción de especies

7. Cambios climáticos: exceso de lluvia e inundaciones, excesivo calor, calentamiento del mar, cambios en la vegetación, modificación de los ciclos biológicos de patógenos

8. Actividades médicas: transfusiones, inyecciones, trasplantes

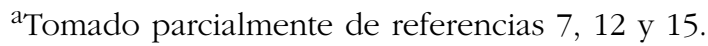


nos de la enfermedad en animales silvestres y domésticos ${ }^{8,12,16}$. La importación de roedores de África como animales regalones introdujo el virus de la viruela de los monos a Estados Unidos de Norteamérica en 2003, produciendo múltiples casos humanos de esta enfermedad desconocida en el país ${ }^{12,16}$. Viajeros humanos diseminaron el virus corona del síndrome respiratorio agudo severo (SARS), desde sus reservorios silvestres en Guangdong, China, a varios países del mundo, incluyendo Canadá28.

FACTORES INTRíNSECOS DEL PATÓGENO QUE FAVORECEN LA EMERGENCIA DE PATÓGENOS ZOONÓTICOS SILVESTRES COMO PATÓGENOS HUMANOS

Respecto de su transmisión a humanos, los patógenos zoonóticos emergentes que tienen un reservorio silvestre pueden dividirse en dos grupos ${ }^{7-9}$. El primero se caracteriza porque en ellos la transmisión del patógeno a humanos es un evento raro, pero una vez producida la subsiguiente transmisión entre humanos mantiene la infección en la población, como ha sucedido con el virus del SIDA y del SARS y, en algunas condiciones con el Hantavirus ${ }^{7-9}$. El segundo grupo está constituido por patógenos que se transmiten directamente o a través de vectores a la población humana y los animales silvestres son siempre el reservorio de la infección, como ocurre con el Hantavirus y la enfermedad de Lyme $8,9,21,22,29,30$. Los patógenos zoonóticos con reservorio silvestre tienen, en general, una gran variedad de medios de transmisión a seres humanos que incluyen transmisión directa, a través de la piel, como sucede con Francisella tularensis ${ }^{4,16}$, por mordedura de animales como el virus de la rabia ${ }^{31}$ y por inhalación de aerosoles contaminados como sucede con los virus de fiebres hemorrágicas y el Hantavirus $^{29,30}$. La ingestión de alimentos contaminados puede también ser un modo de transmisión de zoonosis, como sucede con infecciones por Salmonella, Toxoplasma, Giardia y Cryptosporidium $6,7,16$. Artrópodos son vectores para un número importante de zoonosis emergentes con reservorio silvestre, como ocurre con las garrapatas del genero Ixodes que pueden transmitir la borrelia de la enfermedad de Lyme, Anaplasma, Ehrlichia y el virus de la encefalitis $5,6,14,16$. Simi- larmente, moscas del tipo Glossina e insectos del tipo Triatoma y Reduvidae pueden transmitir tripanosomas en África y en las Américas, respectivamente ${ }^{16,32}$. Los patógenos con un reservorio silvestre habitualmente se caracterizan por tener una población de parásitos que es, generalmente, heterogénea ${ }^{7-9,33-, 35}$. De esta población heterogénea pueden surgir espontáneamente patógenos de una mayor transmisibilidad a humanos y con una virulencia aumentada, facilitando la aparición de epidemias en los individuos susceptibles ${ }^{7-9,33-35}$. Por ejemplo, los reservorios silvestres de $\mathrm{B}$. burgdorferi están infectados con una población heterogénea de este patógeno ${ }^{8,9}$, y en esta población heterogénea existen linajes (clones) que están adaptados para ser transmitidos por garrapatas del tipo Ixodes ${ }^{8,9}$. Una vez que estos diferentes clones son transmitidos a humanos por garrapatas, existen entre ellos algunos más adaptados a diseminarse en mamíferos y a producir de esta manera una infección más severa ${ }^{8,9}$.

Los mecanismos que subyacen estas diferencias son variados e incluyen fenómenos de adaptación a los tejidos del vector y del huésped, la habilidad de evadir la respuesta inmune de éstos y la habilidad de resistir tratamientos terapéuticos y permanecer en forma latente en el huésped ${ }^{8,9}$. Los fenómenos genéticos responsables de esta heterogeneidad en los patógenos zoonóticos son los clásicos de la genética de microorganismos e incluyen la variabilidad genética por mutación espontánea ${ }^{7,35}$ y la recombinación ${ }^{7,34,35}$. La recombinación puede producirse por la infección simultánea de las células de un mismo huésped por patógenos con una constitución genética diferente, como ocurre con los virus de la influenza $\mathrm{A}^{13,20}$. Esta variabilidad también puede ser generada por la transmisión horizontal de la información genética en el huésped o en el ambiente entre patógenos con un patrimonio genético diferente como sucede con los cambios de virulencia y de resistencia a antimicrobianos generados de esta forma en bacterias ${ }^{7,34-36}$. Implícito en la dinámica de los conceptos discutidos está el hecho de que a un mayor tamaño del reservorio silvestre de estos patógenos, existirán mayores posibilidades de su transmisión a la población humana y de generar en ellos variantes con una transmisibilidad y una virulencia aumentada. 
ENFERMEDADES ZOONÓTICAS EN CHILE CON RESERVORIO EN ANIMALES SILVESTRES

En Chile, la clásica enfermedad zoonótica con un reservorio silvestre es la enfermedad de Chagas que es endémica en el centro y norte del territorio nacional ${ }^{37-39}$ (Tabla 3). Trabajos recientes han concluido que la transmisión de esta enfermedad a la población humana infantil ha disminuido drásticamente en el país y que la transmisión por el vector del Trypanosoma cruzi habría sido interrumpida ${ }^{38-}$ 40. Sin embargo, la presencia de un importante reservorio silvestre de la enfermedad en la región endémica y la presencia de los vectores Triatoma y Mepraia con altos niveles de infestación con T cruzi en el ambiente, indica que estos anuncios optimistas deberían ser tomados con cautela ${ }^{38-41}$. Además, trabajos recientes han demostrado el hallazgo de triatomas en un nuevo nicho ecológico asociado a matorrales silvestres, sugiriendo una expansión de estos vectores a un nuevo nicho ${ }^{38}$ y su potencial para transmitir $\mathrm{T}$ cruzi a humanos de manera extradomiciliaria ${ }^{38}$. Cambios climáticos y la explotación forestal de la zona centro-sur del país que podrían resultar en un clima y en un ambiente más seco, podrían producir una expansión del reservorio silvestre y de los vectores de T cruzi hacia la zona sur del país ${ }^{32,41}$. En estas condiciones de expansión del reservorio, aumentos en el número de vectores y la heterogeneidad de las poblaciones de $\mathrm{T}$ cruzi podrían favorecer, también, la aparición de linajes de este protozoo con transmisibilidad y virulencia aumentada ${ }^{10,12,32,41}$.

Cambios climáticos y de la biodiversidad en Chile han resultado en el aumento de los roedores que son el reservorio selvático del Hantavirus $30,31,42,43$. La invasión humana de los biotopos del Hantavirus y la disminución de los predadores de estos roedores, han resultado en la aparición de casos humanos de esta enfermedad en el centro y sur del país $30,31,42,43$. El análisis de la epidemiología de esta enfermedad emergente en el país indica que es una zoonosis de presentación estacional (primavera, verano) con un reservorio silvestre en evolución y que, aparentemente, estaría extendiéndose del sur al norte $30,31,42,43$. Factores de riesgo en la adquisición de esta enfermedad son la actividad al aire libre y habitaciones humanas de calidad deficiente, que permiten la infestación con roedores y la potencial inhalación de aerosoles conteniendo saliva, orina o deposiciones de los roedores infecta$\operatorname{dos}^{30,31,42,43}$. El Hantavirus en Chile es producido por el virus cepa Andes, que produce síndrome

Tabla 3. Ejemplos de zoonosis con reservorio silvestre en $\mathrm{C}$ hile

\begin{tabular}{|lll|}
\hline Zoonosis & Agente & Reservorio Silvestre \\
\hline $\begin{array}{l}\text { Bacterianas } \\
\text { Leptospirosis } \\
\text { Brucelosis }\end{array}$ & Leptospira spp. & \\
& Brucella spp. & Roedores \\
Camélidos \\
Ántrax & Cervidos \\
Tuberculosis & Mycobacterium spp. & Variados \\
& Calmonella spp. & Camélidos \\
Salmonelosis & Cervidos \\
Parasitarias & Trypanosoma cruzi & Roedores \\
Enfermedad de Chagas & Taenia equinococcus & Roedores \\
Hidatidosis & Diphylobothrium latum & Zorros \\
Difilobotriasis & & Salmonideos \\
& & Zorros \\
Virales & Hantavirus & Roedores \\
Fiebre hemorrágica & Virus de la rabia & Murciélagos \\
Rabia & & Roedores \\
& & \\
\hline
\end{tabular}


pulmonar por Hantavirus con una elevada mortalidad, lo que hace la prevención de la enfermedad el arma efectiva de control de la infecciones humanas $30,31,43$. La posibilidad de la transmisión del Hantavirus de humanos a humanos y la imposibilidad de erradicar el reservorio silvestre en aumento, también hacen que la prevención sea el centro del manejo de esta zoonosis ${ }^{42,43}$.

Probablemente, ciertas salmonellosis son también zoonosis con un reservorio silvestre en Chile, a pesar de que no existen estudios detallados de la frecuencia de estos patógenos en animales silvestres en el país ${ }^{44,45}$. Sin embargo, la epidemia de Salmonella Enterica Serovar Enteridis de origen aviario, que Chile experimentó algunos años atrás, probablemente tuvo su origen en roedores domésticos y silvestres que son el reservorio habitual de este patógeno ${ }^{44,45}$. Similarmente, el virus de la rabia debe también en Chile tener un reservorio silvestre, ya que recientemente se describió un caso de rabia humana transmitidos por murciélagos $32,38,47,48$. La presencia de rabia silvestre indica que, en el futuro, este foco pudiera ser el origen de epizootias de rabia en los animales silvestres y domésticos, y éste es un problema que debiera investigarse con acuciosidad para prevenirlo $31,37,46,47$. La presencia y frecuencia de los reservorios silvestres de otras enfermedades zoonóticas, incluyendo brucelosis, ántrax, triquinosis, hidatidosis, influenza A, bartonellosis, lesptopirosis, borreliosis, yersiniosis, toxoplasmosis, giardasis y campilobacteriosis se desconocen y debieran ser investigados, ya que pueden constituir la fuente de futuras epidemias en la población animal y humana secundaria a cambios ambientales ${ }^{37,48-, 50}$ (Tabla 3). La expansión de la acuicultura ha provocado la emergencia de la difilobotriasis, que ha pasado de su histórico nicho silvestre en peces y animales en los lagos de la X Región a los salmónidos cultivados, los que, a su vez se han convertido en fuente de infección humana para este parásito en Chile y en otros países $^{51,52}$. El aumento de la temperaturas del agua de mar como resultado de cambios climáticos, la introducción de la cepa epidémica de Vibrio parahaemolytico O3:K6 al ambiente marino chileno y la alteración de los componentes y ciclos nutricionales en el mar, como resultado de la acuicultura, han disminuido la diversidad biológica de este hábitat y han desencadenado epide- mias de diarrea aguda producidas por este patógeno, además de crear un reservorio de él en las costas de Chile ${ }^{53-56}$. Este breve análisis indica que, al igual que en otras partes del mundo, la emergencia de zoonosis con un reservorio silvestre como causas de enfermedad humana en el país se debe a las interacciones recíprocas entre variados factores ambientales y aquellos intrínsecos del patógeno y de su biología.

¿ES PREVISIBLE LA EMERGENCIA DE ZOONOSIS CON RESERVORIO SILVESTRE COMO CAUSAS DE PATOLOGÍA HUMANA?

Desgraciadamente, a menudo se escucha a científicos y legos en nuestro país que las enfermedades emergentes, incluyendo las zoonosis, son fenómenos naturales. Implícito en el uso de la palabra "natural" está el concepto fatalista de que estas enfermedades serían usuales y que su aparición carecería de causas claras y precisas, haciéndolas de esta manera imprevisibles. Sin embargo, esperamos que nuestra revisión haya convencido a sus lectores que la aparición de las zoonosis con un reservorio silvestre como problemas de salud pública, obedecen a causas antropogénicas y del patógeno y sus vectores, bien establecidas y potencialmente previsibles $^{1,7,12,15,16,58}$ (Tabla 2). En la prevención de estas enfermedades es fundamental la caracterización y el estudio detallado de cada unos de los hábitats silvestres y sus patógenos en el país, y la preservación de la biodiversidad de ellos como fuera sugerido por unos de nosotros (C.C.C.) hace 30 años $1,7,12,15,16,58$. La caracterización de los hábitats silvestres se ha llevado a cabo, clásicamente, con las herramientas de la geografía, de la zoología, de la botánica y de la ecología 1,9,16,32,5961. En años recientes, las herramientas de la biología molecular (reacción en cadena de la polimerasa y la secuenciación de $\mathrm{ADN}$ ) han agregado armas poderosas a la detección de microorganismos de la flora normal y patógena que colonizan e infectan a los animales de vida silvestre en sus hábitats ${ }^{44,45,54,55}$. La eficacia de estas herramientas epidemiológicas moleculares ha sido demostrada en Chile en el estudio de la evolución de infecciones por Salmonella y por $\mathrm{V}$ parahaemolytico ${ }^{44,45,54,55}$. Estas herramientas no sólo permiten detectar la presencia de patógenos 
en animales y vectores y establecer el tamaño del reservorio silvestre, sino que además permiten detectar su heterogeneidad y variabilidad y seguir la dinámica de su evolución, a través de diversos animales y vectores, ayudando a predecir su potencial transmisión a poblaciones humanas susceptibles $8,22,23,44,45,55$.

Chile está enfrentando la degradación acelerada de varios hábitats silvestres y por lo menos dos de ellos ya han sido fuentes de zoonosis: el hábitat boscoso, cuya disminución de biodiversidad e invasión humana ha generado la aparición de infecciones humanas por Hantavirus ${ }^{30,42,43}$ y el hábitat marino, cuya degradación ha facilitado la aparición de enfermedades humanas por vibrios ${ }^{53-}$ 57. Está claro que el empobrecimiento biológico de éstos y otros hábitats silvestres, y los inevitables cambios climáticos que se están desarrollando, le dan cierta urgencia a la formulación de planes para prevenir la aparición de zoonosis emergentes producidas por estos cambios ${ }^{12,19}$. Además, la introducción de patógenos al país, como resultado de aumentos de los viajes y de la actividad comercial, también puede ser otra fuente de zoonosis emergentes que necesitaría ser detectada y controla$\mathrm{da}^{12,14,25}$. La herramienta fundamental de control de las zoonosis es la vigilancia epidemiológica permanente, no sólo de la población humana sino que también de los animales domésticos y silves-

\section{REFERENCIAS}

1. Brown C. Virchow revisited: Emerging zoonoses. ASM News 2003; 69: 493-7.

2. CABELL FC. Enfermedades originadas en el mar. ¿Síntomas del deterioro de la biodiversidad marina de la X Región? Ambientey Desarrollo 2005; 20: 80-7.

3. Cohen ML. Changing patterns of infectious disease. Nature 2000; 406: 762-7.

4. GibBs EPJ. Emerging zoonotic epidemics in the interconnected global community. Veterinary Record 2005; 157: 673-9.

5. Gubler DJ. Resurgent vector-borne diseases as a global health problem. Emerg Infect Dis 1998; 4: 442-50.

6. Krauss H, Weber A, Appel M, Enders B, Isenberg HD, SChiEFer HG et AL. Zoonoses. Infectious Diseases Transmissible from Animals to Humans. Third tres, ya que la detección de casos humanos de una zoonosis generalmente indica un fracaso de los mecanismos preventivos de ellas $4,7,12,14,15,25$. Para esta vigilancia epidemiológica de corte moderno debieran aunarse los esfuerzos y las actividades de individuos e instituciones relacionadas con la salud humana y animal, con el ambiente y su biodiversidad, y con disciplinas como la climatología, la oceanografía y la ecología, entre muchas otras $^{4,7,12,15,25,59,60}$. Indudablemente, planes de este tipo necesitan fondos gubernamentales $y$ privados para el entrenamiento de personal y la instalación de laboratorios para la implementación de las técnicas modernas en el estudio y prevención de estos problemas $4,7,12,15,25,59,60$. Estas actividades son también relevantes para el desarrollo económico del país ya que, por ejemplo Chile, difícilmente podrá ser una potencia alimentaria como ha sido planeado por el gobierno, si no logra controlar la emergencia de zoonosis con reservorio silvestre, capaces de contaminar alimentos consumidos en el país y aquellos de exportación ${ }^{4,7,12,15,25,45,49,53,57,60}$.

\section{Agradecimientos}

La ayuda de las Sras. Betty Barría y Harriett V. Harrison fue invaluable en la preparación del manuscrito. Agradecemos también las excelentes sugerencias de los revisores anónimos.

Edition, Washington, DC: ASM Press, 2003.

7. Weiss RA. The Leeuwenhoek lecture 2001. Animal origins of human infectious diseases. Phil Trans R Soc Lond B 2001; 356: 957-77.

8. Collinge SK, Ray C. Disease Ecology. Community Structure and Pathogen Dynamics. Oxford University Press, 2006.

9. Hudson PJ, Rizzoli A, Grenfell BT, Heesterbeek H, Dobson AP. The Ecology of Wildlife Diseases. Oxford, Great Britain: Oxford University Press, 2002.

10. Pavlovsky EN. Natural Nidality of Transmissible Diseases. In Relation to Landscape Epidemiology of Zooanthroponoses. Peace Publishers. Moscú, 1964.

11. Woolhouse MEJ. Where do emerging pathogens come from? Understanding the origins of pathogens will help us to combat diseases. Microbe 2006; 1: 511. 
12. Daszak P, Cunningham AA, Hyatt AD. Anthropogenic environmental change and the emergence of infectious diseases in wildlife. Acta Tropica 2001; 78: 103-16.

13. Nelson MI, Holmes EC. The evolution of epidemic influenza. Nature Rev 2007; 8: 196-205.

14. Kruse H, Kirkemo A-M, Handeland K. Wildlife as source of zoonotic infections. Emerg Infect Dis 2004; 10: 2067-72.

15. Weiss RA, McMichael AJ. Social and environmental risk factors in the emergence of infectious diseases. Nature Med 2004; 10 (Suppl): S70-S76.

16. Bengis RG, Leighton FA, Fischer JR, Artois M, Mörner T, Tate CM. The role of wildlife in emerging and re-emerging zoonoses. Rev Sci Tech Off Int Epiz 2004; 23: 497-511.

17. Cabello FC, Springer AD. Enfermedades emergentes: antiguas y nuevas enfermedades. Aspectos ecológicos, climáticos e influencias culturales y socio económicas. Rev Méd Chile 1997; 125: 7484.

18. Dobson A, Cattadori I, Holt RD, Ostfeld RS, Keesing F, Krichbaum K eT al. Sacred cows and sympathetic squirrels: The importance of biological diversity to human health. PLoS Med 2006; 3 : e231.

19. Harvell CD, Mitchell CE, Altizer S, Dobson AP, Ostreld RS, Samuel MD. Climate warming and disease risks for terrestrial and marine biota. Science 2002; 296: 2158-62.

20. FAuCI AS. Emerging and re-emerging infectious diseases: Influenza as a prototype of the hostpathogen balancing act. Cell 2006; 124: 665-70.

21. Polley L. Navigating parasite webs and parasite flow: Emerging and re-emerging parasitic zoonoses of wildlife origin. Int J Parasitol 2005; 35: 1279-94.

22. Ostfeld RS, Canham CD, Oggenfuss K, Winchcombe RJ, Keesing F. Climate, deer, rodents, and acorns as determinants of variation in Lyme-disease risk. PLoS Biol 2006; 4: 1058-68.

23. Ostreld RS, Keesing F. Biodiversity and disease risk: the case of Lyme disease. Conserv Biol 2000; 14: 722-8.

24. Fèvre EM, DE C Bronsvoort BM, Hamilton KA, Cleaveland S. Animal movements and the spread of infectious diseases. Trends Microbiol 2006; 14 : 125-31.

25. Karesh WB, Cook RA, Bennett EL, Newcomb J. Wildlife trade and global disease emergence. Emerg Infect Dis 2005; 11: 1000-2.
26. KAHN LH. Confronting zoonoses, linking human and veterinary medicine. Emerg Infect Dis 2006; 12: 556-61.

27. McMichael AJ. Environmental and social influences on emerging infectious diseases: past, present and future. Phil Trans R Soc Lond B 2004; 359: 1049-58.

28. Holmes EC, Rambaut A. Viral evolution and the emergence of SARS coronavirus. Phil Trans R Soc Lond B 2004; 359: 1059-65.

29. Mertz GJ, Hjelle B, Crowley M, Iwamoto G, Tomicic V, VIAL PA. Diagnosis and treatment of new world hantavirus infections. Curr Opin Infect Dis 2006; 19: 437-42.

30. Riguelme R, Riquelme M, Torres A, Rioseco ML, Vergara JA, Scholz L et al. Hantavirus pulmonary syndrome, Southern Chile. Emerg Infect Dis 2003; 9: $1438-43$.

31. Favi M, De Mattos CA, Yung V, Chala E, López LR, De Mattos CC. First case of human rabies in Chile caused by an insectivorous bat virus variant. Emerg Infect Dis 2002; 8: 79-81.

32. Barrett MP, Burchmore RJS, Stich A, Lazzari JO, Frasch AC, Cazzulo JJ ET al. The trypanosomiases. Lancet 2003; 362: 1469-80.

33. READ AF, TAYLOR LH. The ecology of genetically diverse infections. Science 2001; 292: 1099-102.

34. WADE MJ. The co-evolutionary genetics of ecological communities. Nature Rev 2007; 8: 185-95.

35. Woolhouse MEJ. Population biology of emerging and re-emerging pathogens. Trends Microbiol 2002; 10 (Suppl): S3-S7.

36. Cabello FC. Heavy use of prophylactic antibiotics in aquaculture: a growing problem for human and animal health and for the environment. Environ Microbiol 2006; 8: 1137-44.

37. Olea Normandin AM. Zoonosis. Gobierno de Chile. Ministerio de Salud. Bol Vigilancia Salud Pub Chil 2003; 7(19): 39-43.

38. Bacigalupo A, Segura Ja, García A, Hidalgo J, Galuppo S, Cattan PE. Primer hallazgo de vectores de la enfermedad de Chagas asociados a matorrales silvestres en la Región Metropolitana, Chile. Rev Méd Chile 2006; 134: 1230-6.

39. Lorca M, García A, Bahamonde Mi, Fritz A, Tassara R. Certificación serológica de la interrupción de la transmisión vectorial de la enfermedad de Chagas en Chile. Rev Méd Chile 2001; 129: 264-9.

40. Botto-Mahan C, Ortiz S, Rozas M, Cattan PE, SOLARI A. DNA evidence of Trypanosoma cruzi in 
the Chilean wild vector Mepraia spinolai (Hemiptera: Reduviidae). Mem Inst. Oswaldo Cruz. 2005; 100: 237-9.

41. Rozas M, Botto-Maha C, Coronado X, Ortiz S, Cattan PE, Solari A. Short report: Trypanosoma cruzi infection in wild mammals from a chagasic area of Chile. Am J Trp Med Hyg 2005; 73: 517-9.

42. Castillo C, Sanhuzza L, Täger M, Muñoz S, Ossa G, Vial P. Prevalencia de anticuerpos anti-hantavirus en 10 comunas de la Novena Región de Chile donde se han diagnosticado casos de infección clínica por hantavirus. Rev Méd Chile 2002; 130: 251-8.

43. Murúa R, Navarrete M, Cádiz R, Figueroa R, Padula P, ZAROR L et al. Síndrome pulmonar por Hantavirus: situación de los roedores reservorios y la población humana en la Décima Región, Chile. Rev Méd Chile 2003; 131: 169-76.

44. Fernández J, Fica A, Ebensperger G, Calfullan H, Prat S, Fernández A et al. Analysis of molecular epidemiology of Chilean Salmonella enterica serotype enteritidis isolates by pulsed-field gel electrophoresis and bacteriophage typing. J Clin Microbiol 2003; 41: 1617-22.

45. Fica A, Fernández a, Prat S, Figueroa O, Gamboa R, Tsunekawa I ET AL. Salmonella enteritidis, un patógeno emergente en Chile. Rev Méd Chile 1997; 125: 544-51.

46. De Mattos Ca, Favi M, Yung V, Pavietic C, De MatTos CC. Bat rabies in urban centers in Chile. J Wildlife Dis 2000; 36: 231-40.

47. Favi M, Yung V, Pavletic C, Ramírez E, De Mattos C, De Mattos CA. Rol de los murciélagos insectívoros en la transmisión de la rabia en Chile. Arch Med Vet 1999; 31: 157-65.

48. Apt W, Pérez C, Galdamez E, Campano S, Vega F, Vargas D et al. Equinococosis/hidatidosis en la VII Región de Chile: diagnóstico e intervención educativa. Rev Panam Salud Pública 2000; 7: 8-16.

49. Drancourt M, Houhamdi L, Raoult D. Yersinia pestis as a telluric, human ectoparasite-borne organism. Lancet Infect Dis 2006; 6: 234-41.
50. McBride AJA, Athanazio DA, Reis MG, KO AI. Leptospirosis. Curr Opin Infect Dis 2005; 18: 37686.

51. CABELLO FC. Salmon aquaculture and transmission of the fish tapeworm. Emerg Infect Dis 2007; 13: 169-71.

52. Cabello FC. Acuicultura y salud pública. La expansión de la difilobotriasis en Chile y mundo. Rev Méd Chile 2007; 135: 1064-71.

53. Cabello FC, Espejo RT, Hernández MC, Rioseco ML, Ulloa J, Vergara JA. Vibrio parahaemolyticus O3:K6 epidemic diarrhea, Chile, 2005. Emerg Infect Dis 2007; 13: 655-6.

54. González-Escalona N, Cachicas V, Acevedo C, Rioseco Ml, Vergara Ja, Cabello F et al. Vibrio parahaemolyticus diarrhea, Chile, 1998 and 2004. Emerg Infect Dis 2005; 11: 129-31.

55. Fuenzalida l, Armijo l, Zabala B, Hernández C, Rioseco ML, Riquelme C et al. Vibrio parahaemolyticus strains isolated during investigation of summer 2006 seafood related diarrhea outbreaks in two regions of Chile. Int J Food Microbiol 2007; 117: $270-5$.

56. Hernández C, Ulloa J, Vergara Ja, Espejo R, Cabello FC. Infecciones por Vibrio parahaemolyticus e intoxicaciones por algas: problemas emergentes de salud pública en Chile. Rev Méd Chile 2005; 133: 1081-8.

57. CABELLO C. Consideraciones sobre la importancia del estudio de las enfermedades de los animales silvestres. Rev Soc Med Vet Chil 1974; 24: 24-8.

58. Rogers DJ, Randolph SE. Studying the global distribution of infectious diseases using GIS and RS. Nature Rev Microbiol 2003; 1: 231-7.

59. Smith KF, Dobson AP, McKenzie Fe, Real LA, Smith DL, WILSON ML. Ecological theory to enhance infectious disease control and public health policy. Front Ecol Environ 2005; 3: 29-37.

60. Zinsstag J, Schelling E, Roth F, Bonfoh B, De Savigny D, Tanner M. Human benefits of animal interventions for zoonosis control. Emerg Infect Dis 2007; 13: $527-31$. 